\title{
Radiographic Evaluation of Surgical Treatment of Peri-implantitis with Bone Graft Procedure: Case Report
}

\author{
Bo-Ram Nam, DDS', Yeon-Tae Kim, DDS, MSD², Jae-Hong Lee, DDS, MSD, PhD ${ }^{3,4}$, \\ Seong-Nyum Jeong, DDS, MSD, $\mathrm{PhD}^{3,4 *}$ \\ ${ }^{1}$ Resident, Department of Periodontology, Daejeon Dental Hospital, College of Dentistry, Wonkwang University, \\ Daejeon, Korea \\ ${ }^{2}$ Fellow, Department of Periodontology, Daejeon Dental Hospital, College of Dentistry, Wonkwang University, \\ Daejeon, Korea \\ ${ }^{3}$ Professor, Department of Periodontology, Daejeon Dental Hospital, College of Dentistry, Wonkwang \\ University, Daejeon, Korea \\ ${ }^{4}$ Professor, Institute of Wonkwang Dental Research, Wonkwang University College of Dentistry, Iksan, Korea \\ *Corresponding author: Seong-Nyum Jeong, Professor, Department of Periodontology, Wonkwang \\ University Daejeon Dental Hospital, Wonkwang University School of Dentistry, 77 Dunsan-ro, Seo-gu, \\ Daejeon 35233, Korea. \\ Tel: +82-42-366-1141. Fax: +82-42-366-1115. E-mail: seongnyum@wku.ac.kr
}

\section{OPEN ACCESS}

pISSN 1229-5418

eISSN 2671-6623

Implantology 2020; 24(3): 127-136

https://doi.org/10.32542/implantology.202013

Received: May 7, 2020

Revised: June 25, 2020

Accepted: July 11, 2020

ORCID

Bo-Ram Nam

https://orcid.org/0000-0001-5708-9956

Yeon-Tae Kim

https://orcid.org/0000-0001-7209-3208

Jae-Hong Lee

https://orcid.org/0000-0002-2375-0141

Seong-Nyum Jeong

https://orcid.org/0000-0003-4890-989X

Copyright $($ 2020. The Korean Academy of Oral \& Maxillofacial Implantology

\footnotetext{
This is an Open Access article distributed under the terms of the Creative Commons Attribution Non-Commercial License (http://creativecommons. org/licenses/by-nc/4.0/) which permits unrestricted non-commercial use, distribution, and reproduction in any medium, provided the original work is properly cited.
}

\section{Abstract}

The purpose of this case report was to radiographically evaluate the long-term effect of surgical treatment of peri-implantitis defects using bone graft procedure for more than 3 years after operation. 2 cases of patients who had peri-implantitis induced intrabony defect were included in this study. Patients were treated by surgical procedure which consists of mechanical debridement with air abrasive system and titanium curette, chemical decontamination with tetracycline, and regenerative approach using synthetic bone or xenogenic bone. Clinical reduction of probing pocket depth and radiographic evidence of bone gain was observed and maintained in both patients for 3 years after surgery. Within the limitation of this case report, bony defects were managed effectively by surgical treatment with regenerative bone graft procedure.

Keywords: Bone Regeneration, Bone Substitutes, Peri-Implantitis, Surgical Procedures

\section{I . Introduction}

Dental implant therapy is a well-established method to replace missing teeth. ${ }^{1}$ However, various types of failure and adverse effects are observed with popular use of implants. Peri-implant diseases including peri-implant mucositis and peri-implantitis have become one of the most frequent complications affecting the tissues around implants which can lead to the loss of the implants. ${ }^{2,3}$ It is generally accepted that the microbial biofilm plays an analogous role in the development of peri-implant inflammation as colonization 
follows a similar principle at teeth and implant surface. ${ }^{3,4}$ Despite limited data, peri-implant mucositis and peri-implantitis have been reported to affect $50 \%$ and $12-43 \%$ of the implants, respectively. Clinically, peri-implantitis sites exhibit signs of inflammation, bleeding on probing (BOP), and/or suppuration, increased probing pocket depths (PPD), and/or recession of the mucosal margin. Also, bone loss is shown radiographically compared to previous examinations, unlike peri-implant mucositis, which affects only soft tissue around implant.,

Various non-surgical and surgical treatment have been introduced for the control of peri-implant disease. Non-surgical treatment for peri-implant mucositis is effective for reducing inflammation of peri-implant tissue. ${ }^{3-5}$ However, for peri-implantitis, non-surgical treatment showed limited improvements in the main clinical parameters and there was a tendency to recurrence of the disease because of insufficient implant surface disinfection. ${ }^{5}$ Generally, surgical treatment includes mechanical debridement, ${ }^{2,6}$ chemical decontamination, ${ }^{2,7}$ local administration of antibiotics, ${ }^{8}$ and regenerative procedure using bone graft., ${ }^{5,9}$ These can be applied simultaneously under open flaps and/or done in multiple combinations. In a retrospective study by Langervall et al., ${ }^{10}$ it was shown that $84 \%$ of the open flap debridement had been successful at implant level.

Surgical treatment with regenerative procedure using bone graft shows statistically significant improvements in radiographic bone fill compared to open flap debridement alone. ${ }^{11}$ Although a partial defect fill can be expected, but complete fill of intrabony defects caused by peri-implantitis using a guided bone regeneration (GBR) seems not to be a predictable outcome. ${ }^{12}$

Most studies of peri-implantitis treatment reported only short-term results. Also reference for domestic condition is hard to find. Therefore, the purpose of this case report was to radiographically evaluate the long-term effect of surgical treatment of peri-implantitis defects using regenerative bone graft procedure for more than 3 years after operation.

\section{Case}

\section{Case 1}

A 71-year-old male patient visited the department of periodontology because of implant loss and peri-implantitis on left posterior mandible. He had received implant surgery on \#35, \#36 3 years ago, but had been suffering from swelling and pus around implants for 2 years, and finally lost \#36 implant spontaneously the day before visiting the hospital.

Probing pocket depth (PPD) and radiographic bone defect depth was measured at baseline and over 3 
years after surgery. Marginal bone defect was measured via distance of implant fixture-abutment interface (IP) to most coronal point of bone to implant contact (BIC) (IP-BIC).

A $6 \mathrm{~mm}$ PPD with $1 \mathrm{~mm}$ peri-implant mucosal recession was observed at the lingual side of \#35 implant (\#i35). Additionally, BOP and pus discharge were observed. Implant mobility was not observed. Marginal bone defect of $6 \mathrm{~mm}$ depth from IP was observed at mesial site of \#35 implant, and large radiolucent lesion due to loss of \#36 implant was detected on the baseline panoramic radiograph (Fig. 1).
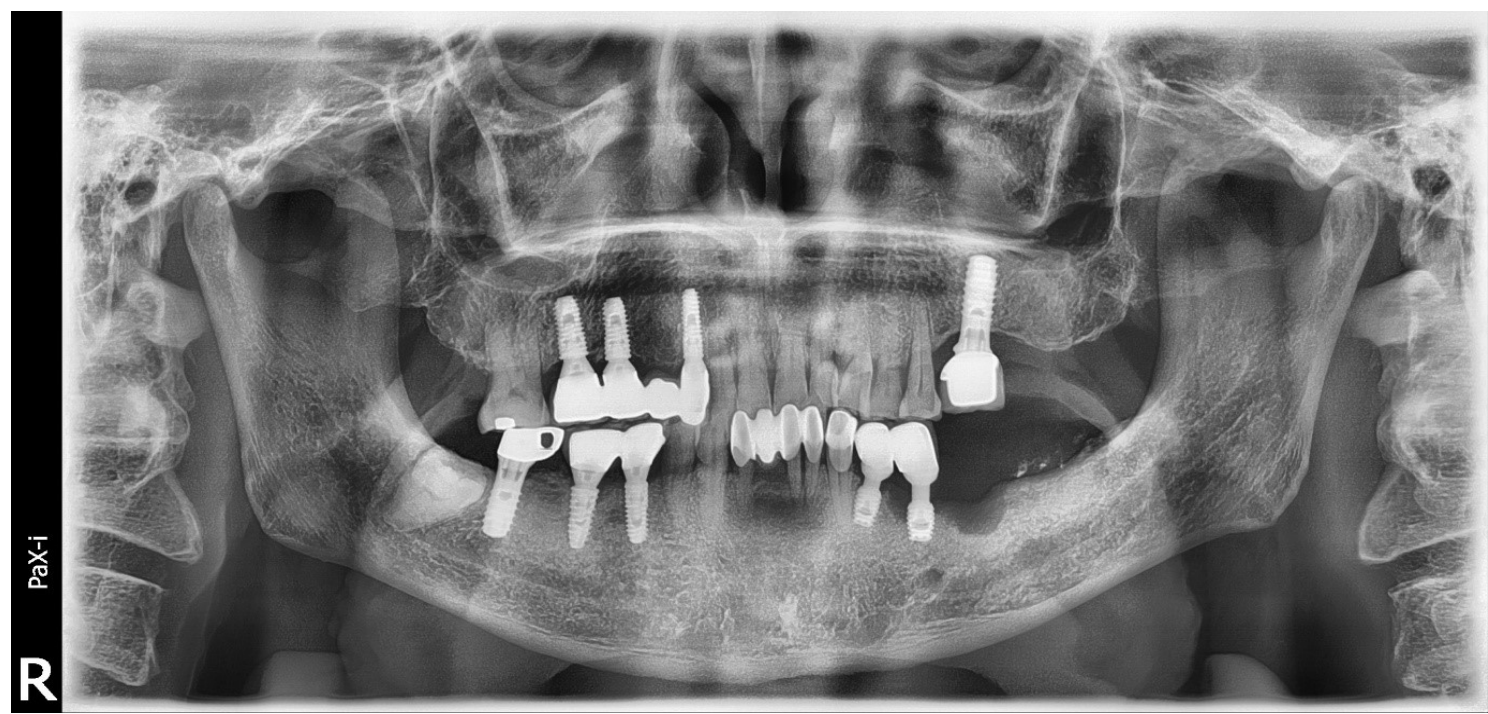

Fig. 1. First visit panoramic image of case 1 patient. Around \#35 implant, a circumferential radiolucent lesion up to $6 \mathrm{~mm}$ below the implant shoulder was shown.

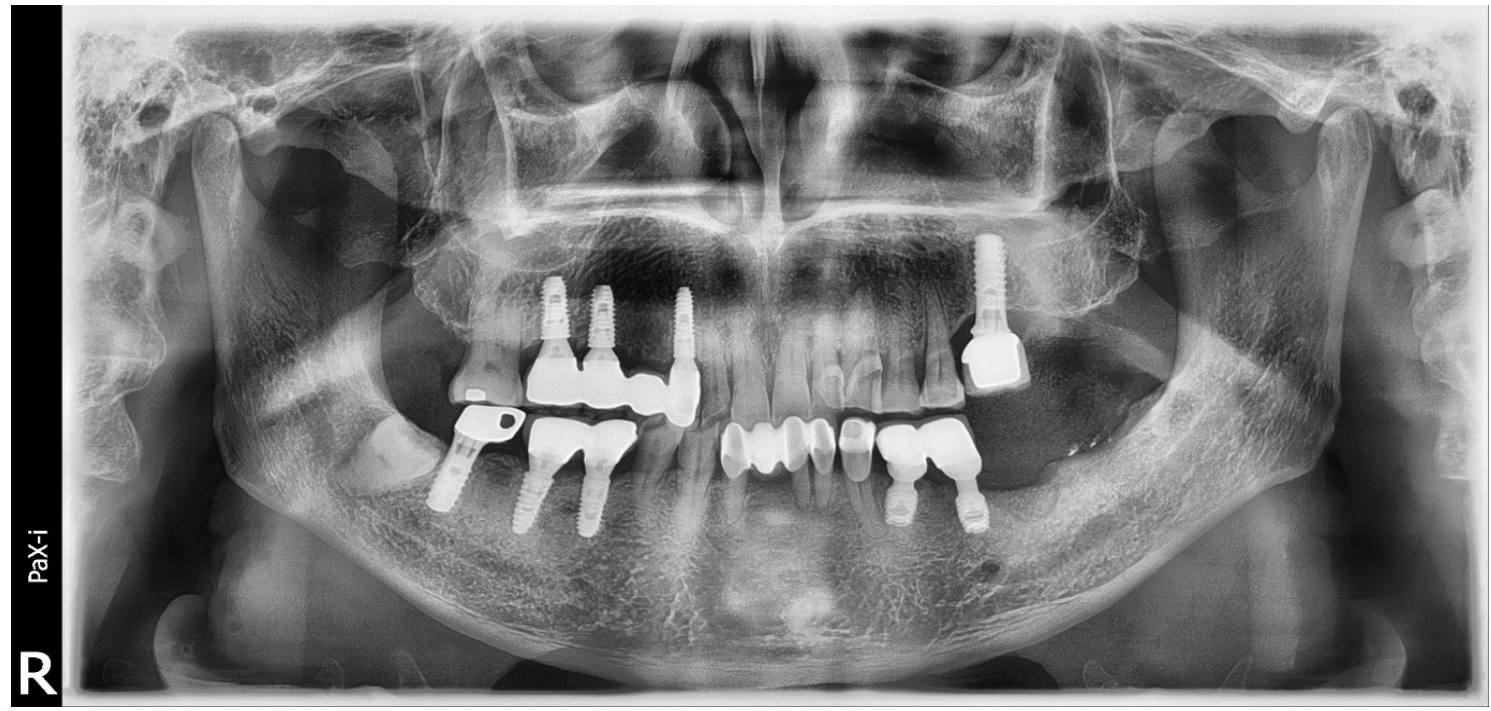

Fig. 2. Panoramic image of case 1 patient immediately after surgery. The defects were filled by graft material well. 
At first visit, we performed non-surgical therapy including scaling, and prescription of antibiotics and anti-inflammatory drugs. We scheduled a surgery to treat a peri-implantitis lesion and bone defect due to implant loss of \#36 2 weeks after initial therapy. After the local anesthesia with $2 \%$ lidocaine HCL with 1:100,000 epinephrine, intra-sulcular incision was made at the surgical site with blade and interdental knife (Hu-Friedy, Chicago, IL, USA). Mechanical debridement with air-abrasive system (Prophyflex3, KaVo Dental, Biberach, Germany), titanium ultrasonic scaler, and adjunctive chemical decontamination with tetracycline (TC) cotton and solution were performed. On the circumferential
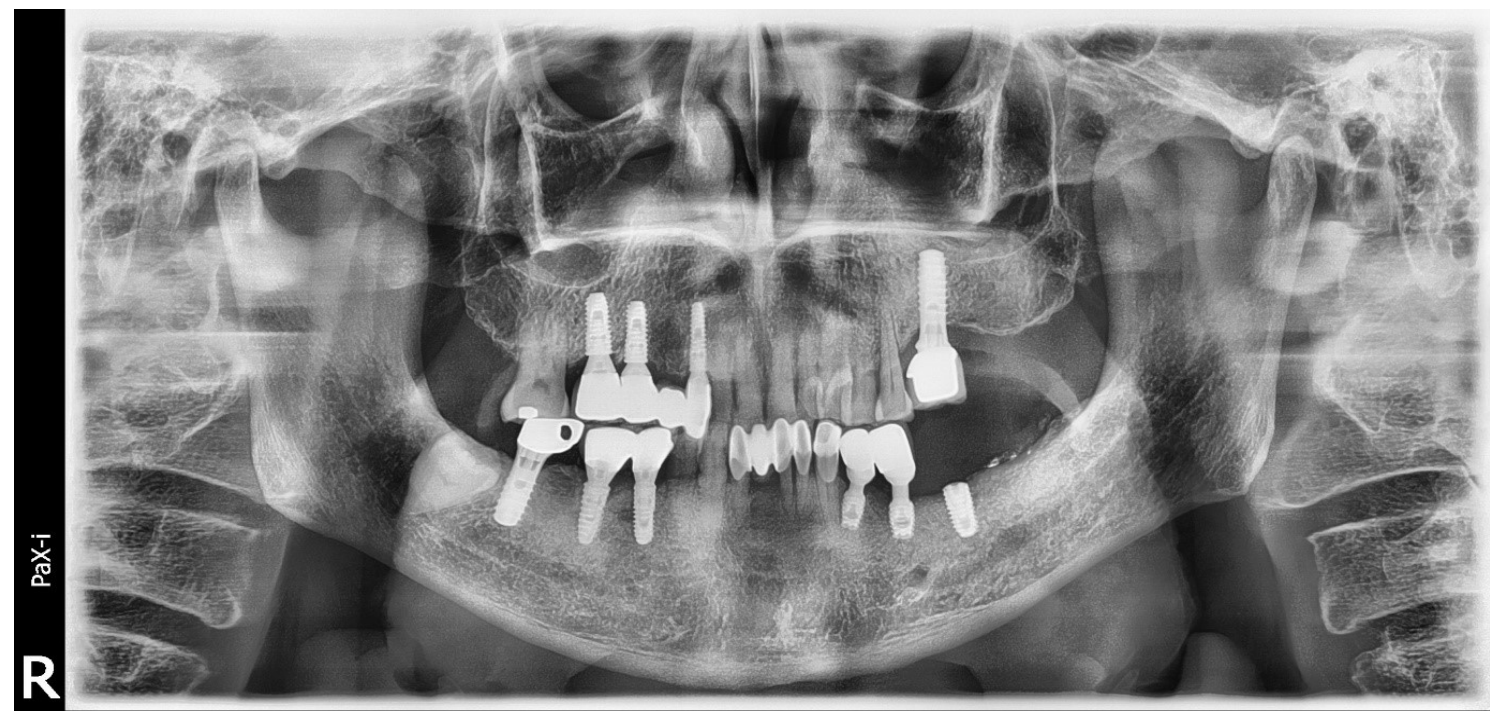

Fig. 3. After 6 months follow up, implant was installed on \#36. The graft material on \#i35 was well remained.

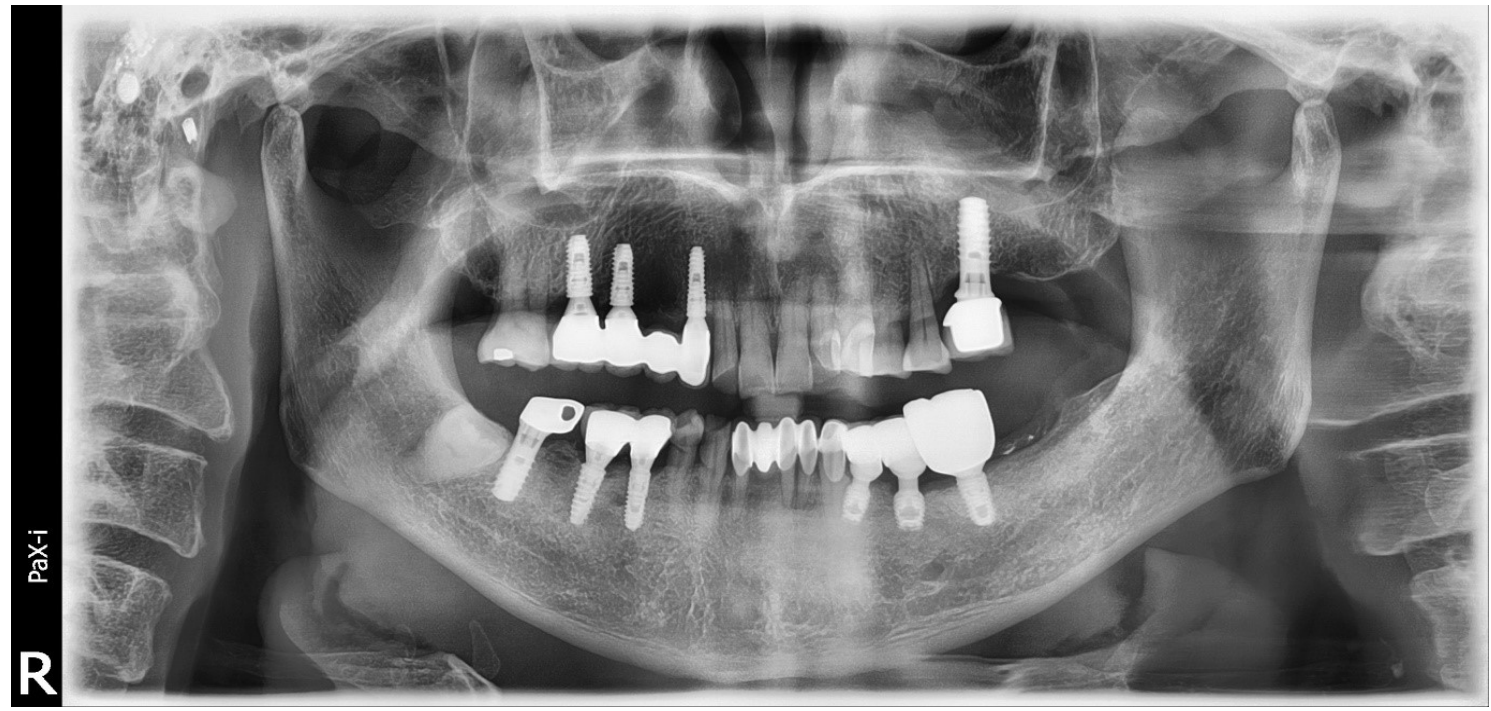

Fig. 4. After 3 years follow up, radiographic improvement of $5 \mathrm{~mm}$ on \#i35 was observed. \#36 implant funtioned normally. 
defect of \#i35, bone graft was done with synthetic bone (MBCP, Biomatlante, Vigneux, France). Guided bone regeneration was tried with MBCP and collagen membrane (Lyoplant ${ }^{\circledR}$, Braun Aesculap, Tutsingen, Germany) on \#36 explantation defect. The surgical site was sutured with 4-0 e-PTFE (Biotex ${ }^{\circledR}$, Purgo biologics, Seongnam, South Korea) using modified horizontal mattress and interrupted suture method. A panoramic radiograph was taken immediately after surgery (Fig. 2). 2 weeks after surgery, the stitch out was done. The patient's chief complaint including pain and pus discharge was resolved. After 6months of bone graft, we performed first stage implant surgery of \#36 (Fig. 3). The grafted MBCP material on the lingual defect of \#i35 seemed to be normal bone tissue with naked eye on the first stage implant surgery. The PPD was reduced to $2 \mathrm{~mm}$, and radiographic improvement of $5 \mathrm{~mm}$ defect fill was observed after 3 years after surgery (Fig. 4).

\section{Case 2}

A 50-year-old male patient visited the department of periodontology with discomfort in the right posterior mandible. He received implant surgery on right mandibular second premolar 2 years ago. There we could observe PPD deeper than $10 \mathrm{~mm}$ with pus discharge, and peri-implant mucosal swelling. On the panoramic and periapical image, we could detect severe circumferential bone loss around \#45 implant (\#i45). Marginal bone defect was measured via distance of implant fixture-abutment interface (IP) to most coronal point of bone to implant contact (BIC). The deepest bone defect was detected at distolingual side of \#i45 showing a depth of $7 \mathrm{~mm}$. Also, apical lesion of \#44 treated with post and crown was detected. Because \#44 had no specific symptoms and he didn't want to treat the lesion, we decided to watch the periapical lesion periodically (Fig. 5).

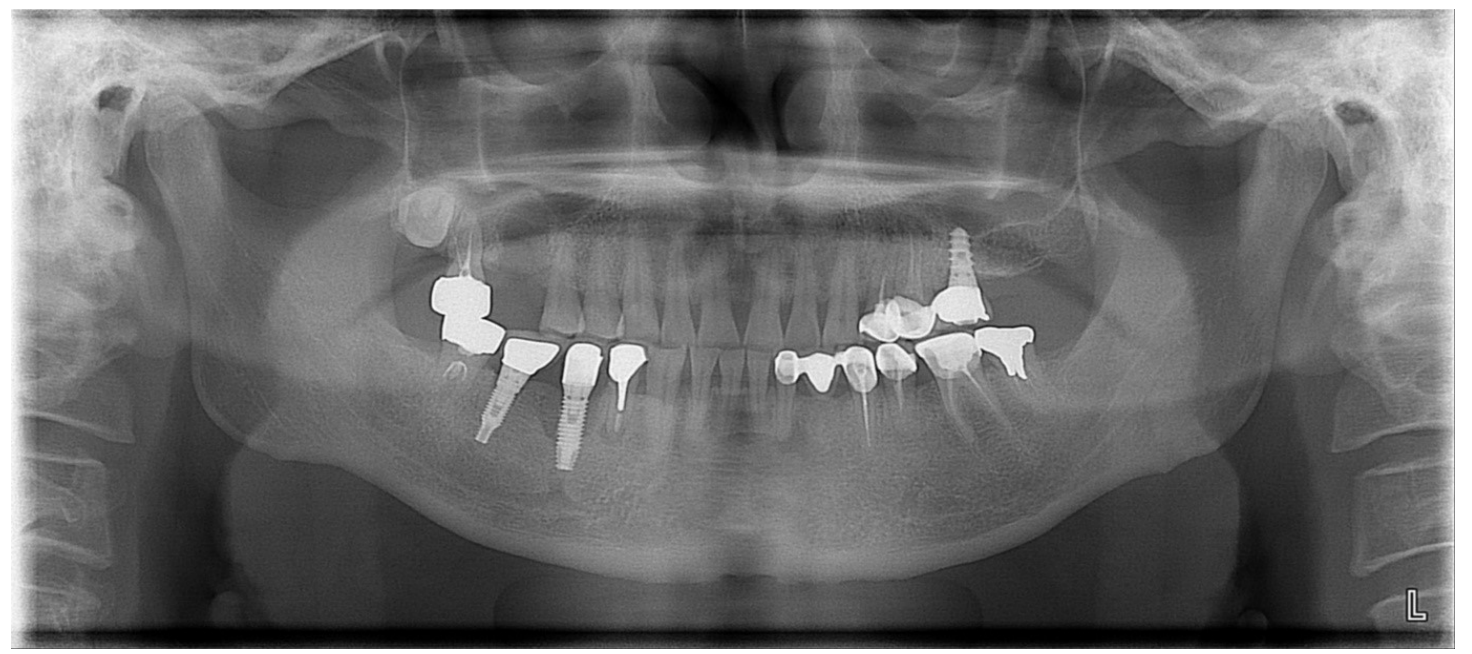

Fig. 5. First visit panoramic image of case 2 patient. There was a $7 \mathrm{~mm}$ mesio-disto lingual intrabony defect on \#45 implant. 
At 3 months after non-surgical treatment, peri-implantitis surgery was provided for the patient because a suppuration recurred on the defect. On incision and flap elevation, \#i45 showed severe lingual bone loss. Mechanical debridement with air-abrasive system (Prophyflex3, KaVo Dental, Biberach, Germany), ultrasonic scaler, and adjunctive chemical decontamination with TC cotton and solution was done. After mechanical and chemical decontamination procedure on \#i45, xenogenic bone (OCS- ${ }^{\circledR}$, NIBEC, Seoul, Korea) was filled in the circumferential defect. Surgical site was sutured with 4-0 e-PTFE (Biotex ${ }^{\circledR}$, Purgo biologics, Seongnam, South Korea) and 5-0 nylon (Monosof ${ }^{\circledR}$, Covidien, $^{-}$

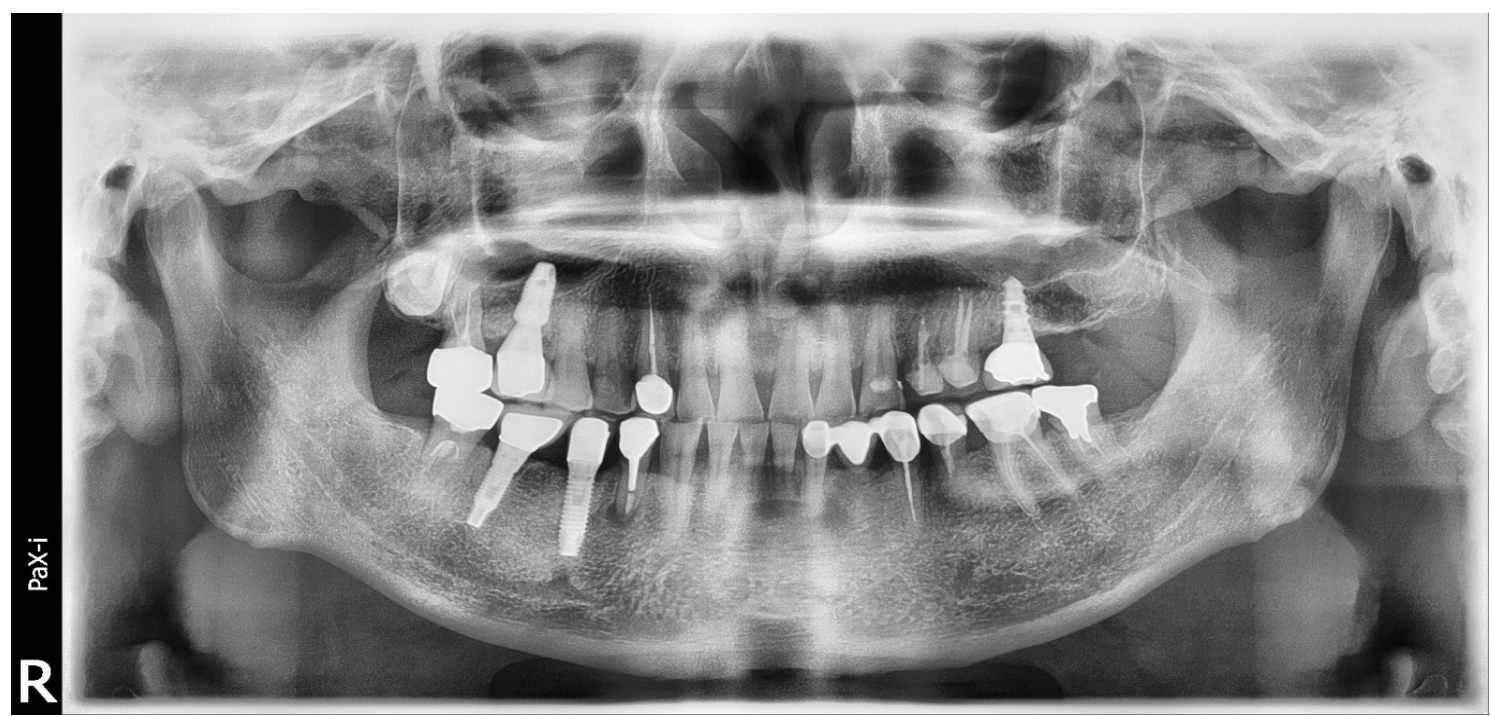

Fig. 6. The radiograph taken after 1-year follow-up. Operation site remained stable. Radiographically, bone defect decreased to $3 \mathrm{~mm}$.

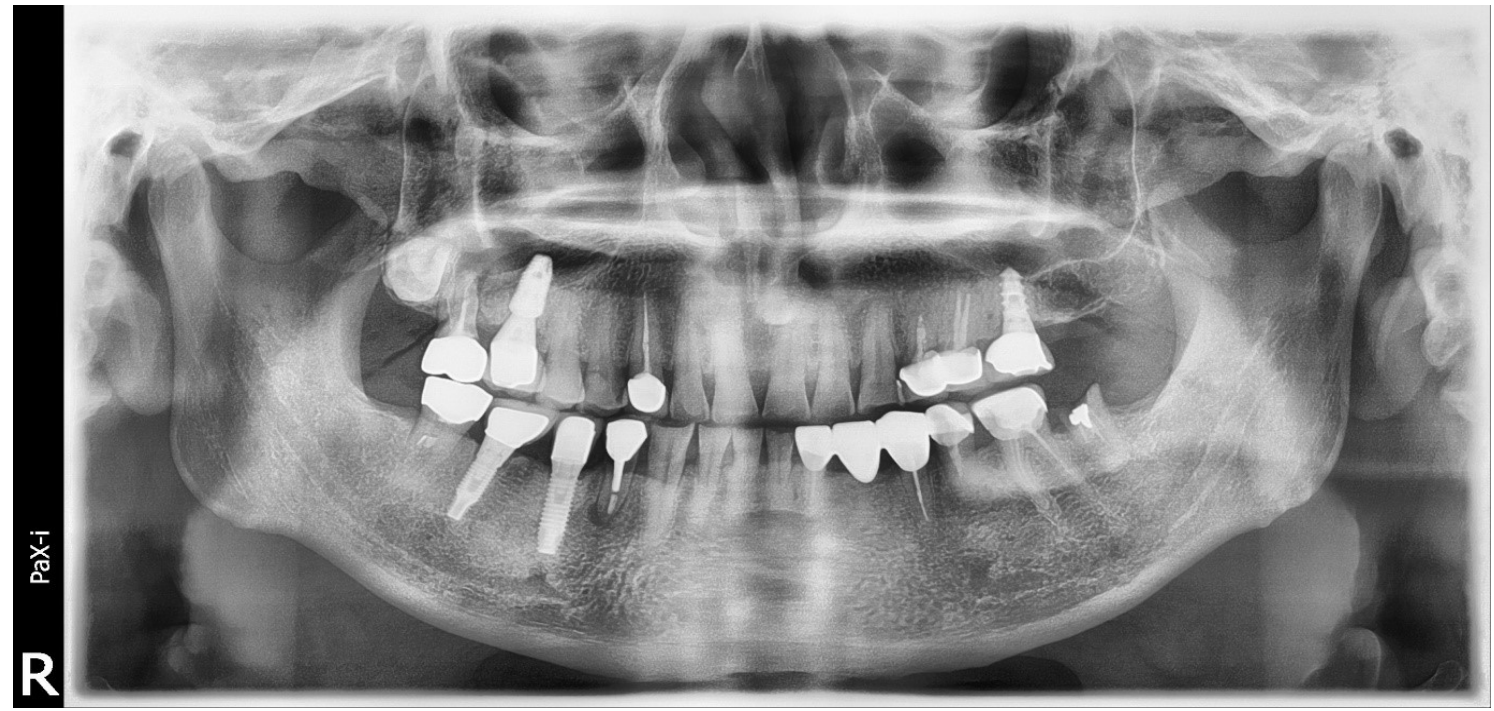

Fig. 7. After 3 years follow-up, panoramic image proved well remained bone fill. He lost a crown of \#37. 
Dublin, Ireland) using modified horizontal mattress and interrupted suture method. 2 weeks after surgery, the stitch out was done, and the patient's discomfort of swelling and pain disappeared. At 1-year follow-up, the PPD was reduced to $5 \mathrm{~mm}$ and the operation site remained stable. Radiographically the bone defect decreased to $3 \mathrm{~mm}$ (Fig. 6). Bone defect was filled $4 \mathrm{~mm}$ compared to baseline. Up to 3 years follow-up, panoramic image showed bone fill stably, and apical lesion of \#44 remained stable (Fig. 7).

\section{Results}

Clinical and radiographic improvements were observed in follow-up after treatment. Clinical reduction of PPD and radiographic evidence of bone gain was observed in all 2 patients. Changes in PPD showed reductions of $4 \mathrm{~mm}, 5 \mathrm{~mm}$ in case 1 and 2 respectively. Radiographic bone defect depths decreased by $5 \mathrm{~mm}, 4 \mathrm{~mm}$ respectively, which remained for 3 years (Table 1).

Table 1. Preoperative and postoperative probing pocket depth (PPD) and radiographic bone defect depth

\begin{tabular}{lccccccc} 
& \multicolumn{3}{c}{ PPD } & & & \multicolumn{3}{c}{ Radiographic bone defect } \\
\cline { 2 - 3 } \cline { 7 - 8 } Case 1 & Preoperative & Postoperative & $\begin{array}{c}\text { Amount of } \\
\text { change }\end{array}$ & & Preoperative & Postoperative & $\begin{array}{c}\text { Amount of } \\
\text { change }\end{array}$ \\
Case 2 & $6 \mathrm{~mm}$ & $2 \mathrm{~mm}$ & $4 \mathrm{~mm}$ & & $6 \mathrm{~mm}$ & $1 \mathrm{~mm}$ & $5 \mathrm{~mm}$ \\
Mean $\pm \mathrm{SD}(\mathrm{mm})$ & $10 \mathrm{~mm}$ & $5 \mathrm{~mm}$ & $5 \mathrm{~mm}$ & & $7 \mathrm{~mm}$ & $3 \mathrm{~mm}$ & $4 \mathrm{~mm}$ \\
\hline
\end{tabular}

\section{Discussion}

Several studies reported that surgical treatment with regenerative procedure using bone graft showed statistically significant improvements in radiographic bone fill compared to open flap debridement alone. ${ }^{11}$ However, there are few studies in Korea that have reported long-term effect of bone graft on peri-implantitis defect. The purpose of this case report was to radiographically evaluate the long-term effect of surgical treatment of peri-implantitis defects using regenerative bone graft procedure for more than 3 years. Clinical reduction of probing pocket depth and radiographic evidence of bone gain was observed and maintained in both patients 3 years after surgery.

There were many studies reporting that non-surgical therapy is effective for reducing inflammation of peri-implant tissue for peri-mucositis but not for peri-implantitis lesion. ${ }^{3-5}$ There was a tendency to recurrence of the disease because of insufficient implant surface disinfection., ${ }^{5,}$ Decontamination protocols such as mechanical debridement with air-abrasive system, titanium or plastic curette and 
ultrasonic scaler were effective for removal of biofilm. ${ }^{2,6}$ However using only mechanical method often caused incomplete surface disinfection because of instrument accessibility. ${ }^{6}$ Therefore, adjunctive chemical decontamination agents including citric acid, tetracycline, chlorohexidine, acidified phenolics, and ethylenediaminetetraacetic acid (EDTA) are commonly used to treat inaccessible area of implant. ${ }^{2,7}$ Although many mechanical and chemical decontamination methods were tried clinically, no definite gold standard has been identified. ${ }^{13}$ Tetracycline have been used with bone graft material in regenerative surgical techniques, due to anti-microbial effect, anti-collagenase, and positive effect with bone graft. ${ }^{14}$ ${ }^{15}$ In a review by Javed et al., ${ }^{16}$ systemic and local antibiotic applications led to significant reductions of probing pocket depths in a period between one and six year. Moreover, it was shown that the decontamination procedure using tetracycline followed by guided bone regeneration resulted in arrest of inflammation and radiographic bone fill., ${ }^{7,15} \mathrm{TC}$ cotton and solution was used in the decontamination process in this study. However, it was not possible to confirm the effect of TC alone in this study. More sophisticatedly designed studies are needed to demonstrate the beneficial effect of TC conditioning alone, excluding the effect of bone graft.

Following decontamination of the implant surface, regenerative techniques have been introduced to regenerate the bone loss due to infection in an attempt to achieve re-osseointegration of implant surface. ${ }^{17}$ Regenerative procedures have two main objectives: to support the tissue dimensions during the healing process, avoiding recession of the mucosa, and to enhance the chance of obtaining reosseointegration, using reconstructive and regenerative techniques/materials. ${ }^{5}$ So far, there doesn't seem to be much evidence of which surgical method is superior to the other.

Regarding to grafting materials, autogenous, allogenic, synthetic and xenogenic bone replacement materials were often used for augmentation in bone defects with or without collagen membrane. It seemed allogenic and xenogenic grafts might be almost equivalent to autogenous material. ${ }^{18,19}$ In the case of this study, synthetic bone and xenogenic bone were used, and it was found to be effective for defect fill regardless of the type of graft materials, which maintained up to 3 years. However, the factors that could affect the outcomes, such as the type of bone defects, the degree of defects, the type of graft materials, and adjacent teeth or implant types were not controlled in this study, so further studies are needed to evaluate the effects of each factor.

In general, regenerative procedure using membrane or bone graft material alone have been shown to be more effective than open flap debridement alone regarding to bone regeneration and reosseointegration of peri-implantitis defect. ${ }^{13}$ Also several studies reported the effects of combined use of membranes and bone graft materials were superior to those using membranes or bone grafts alone and 
tend to give the best results. ${ }^{13,20}$ However, there is a high variability in the amount of bone fill due to different investigation protocols and measurement. In previous studies of surgical augmentative treatment of peri-implantitis, PPD reduction in the range of 0.74 to $5.4 \mathrm{~mm}$ and radiographic bone fill of $57 \%$ to $93.3 \%$, and vertical defect reduction varied from 0.2 to $3.77 \mathrm{~mm}$ was reported. ${ }^{11}$ Patients in this study showed a 4-5 mm improvement in radiographic bone defect and PPD. However, the results should be carefully interpreted as it has not been confirmed to be a true histological regeneration.

\section{Conclusion}

In this study, we used regenerative surgical technique, using synthetic graft with absorbable membrane or xenograft without membrane, with mechanical and chemical decontamination to the defect showing circumferential bone loss and intrabony defect. Within the limitation of this case report, bone defects were managed effectively by surgical treatment with regenerative bone graft procedure. Further studies with long term follow up and controlled factor should be needed for treatment protocol of periimplantitis defect.

\section{Acknowledgement}

This study was supported by Wonkwang University in 2020.

\section{References}

1. Simonis P, Dufour T, Tenenbaum H. Long-term implant survival and success: a 10-16-year followup of non-submerged dental implants. Clin Oral Implants Res 2010;21:772-7.

2. Smeets R, Henningsen A, Jung O, Heiland M, Hammacher C, Stein JM. Definition, etiology, prevention and treatment of peri-implantitis--a review. Head Face Med 2014;10:34.

3. Berglundh T, Armitage G, Araujo MG, Avila-Ortiz G, Blanco J, Camargo PM, et al. Peri-implant diseases and conditions: Consensus report of workgroup 4 of the 2017 World Workshop on the Classification of Periodontal and Peri-Implant Diseases and Conditions. J Periodontol 2018;89 Suppl 1:S313-S8.

4. Renvert S, Persson GR, Pirih FQ, Camargo PM. Peri-implant health, peri-implant mucositis, and peri-implantitis: Case definitions and diagnostic considerations. J Periodontol 2018;45 Suppl 20: S278-S85.

5. Figuero E, Graziani F, Sanz I, Herrera D, Sanz M. Management of peri-implant mucositis and periimplantitis. Periodontol 2000 2014;66:255-73.

6. Ronay V, Merlini A, Attin T, Schmidlin PR, Sahrmann P. In vitro cleaning potential of three implant debridement methods. Simulation of the non-surgical approach. Clin Oral Implants Res 2017;28:151-5. 
7. Lopez MA, Andreasi Bassi M, Confalone L, Silvestre F, Arcuri C. The treatment of peri-implant diseases: a new approach using hyben $x^{\circledR}$ as a decontaminant for implant surface and oral tissues. Oral Implantol (Rome) 2016;9:106-14.

8. Cha JK, Lee JS, Kim CS. Surgical Therapy of Peri-Implantitis with Local Minocycline: A 6-Month Randomized Controlled Clinical Trial. J Dent Res 2019;98:288-95.

9. Kim S, Hu KS, Jung UW. Reosseointegration After Regenerative Surgical Therapy Using a Synthetic Bone Substitute for Peri-implantitis: Human Autopsy Study. Int J Periodontics Restorative Dent 2018;38:585-91.

10. Lagervall M, Jansson LE. Treatment outcome in patients with peri-implantitis in a periodontal clinic: a retrospective study. J Periodontol 2013;84:1365-73.

11. Ramanauskaite A, Obreja K, Sader R, Khoury F, Romanos G, Koo KT, et al. Surgical Treatment of Periimplantitis With Augmentative Techniques. Implant Dent 2019;28:187-209.

12. Sahrmann P, Attin T, Schmidlin PR. Regenerative treatment of peri-implantitis using bone substitutes and membrane: a systematic review. Clin Implant Dent Relat Res 2011;13:46-57.

13. Claffey N, Clarke E, Polyzois I, Renvert S. Surgical treatment of peri-implantitis. J Clin Periodontol 2008;35:316-32.

14. Park JB. Treatment of peri-implantitis with deproteinised bovine bone and tetracycline: a case report. Gerodontology 2012;29:145-9.

15. Tinti C, Parma-Benfenati S. Treatment of peri-implant defects with the vertical ridge augmentation procedure: a patient report. Int J Oral Maxillofac Implants 2001;16:572-7.

16. Javed F, Alghamdi AS, Ahmed A, Mikami T, Ahmed HB, Tenenbaum HC. Clinical efficacy of antibiotics in the treatment of peri-implantitis. Int Dent J 2013;63:169-76.

17. Renvert S, Polyzois I, Maguire R. Re-osseointegration on previously contaminated surfaces: a systematic review. Clin Oral Implants Res 2009;20:216-27.

18. Kolk A, Handschel J, Drescher W, Rothamel D, Kloss F, Blessmann M, et al. Current trends and future perspectives of bone substitute materials - from space holders to innovative biomaterials. $\mathrm{J}$ Craniomaxillofac Surg 2012;40:706-18.

19. Fischer J, Kolk A, Wolfart S, Pautke C, Warnke PH, Plank C, et al. Future of local bone regeneration - Protein versus gene therapy. J Craniomaxillofac Surg 2011;39:54-64.

20. Machado MA, Stefani CM, Sallum EA, Sallum AW, Tramontina VA, Nociti Junior FH. Treatment of ligature-induced peri-implantitis defects by regenerative procedures: a clinical study in dogs. J Oral Sci 1999;41:181-5. 